\title{
The influence of bicycle lean on maximal power output during sprint cycling
}

\author{
Ross D. Wilkinson*, Rodger Kram \\ Locomotion Laboratory, Department of Integrative Physiology, University of Colorado, Boulder, CO, \\ $U S A$
}

\begin{abstract}
Competitive cyclists typically sprint out of the saddle and alternately lean their bikes from side-to-side, away from the downstroke pedal. Yet, there is no direct evidence as to whether leaning the bicycle, or conversely, attempting to minimize lean, affects maximal power output during sprint cycling. Here, we modified a cycling ergometer so that it can lean from side-to-side but can also be locked to prevent lean. This modified ergometer made it possible to compare maximal 1-s crank power during non-seated, sprint cycling under three different conditions: locked (no lean), ad libitum lean, and minimal lean. We found that leaning the ergometer ad libitum did not enhance maximal 1-s crank power compared to a locked condition. However, trying to minimize ergometer lean decreased maximal 1-s crank power by an average of $5 \%$ compared to leaning ad libitum. IMU-derived measures of ergometer lean provided evidence that, on average, during the $a d$-lib condition, subjects leaned the ergometer away from the downstroke pedal as in overground cycling. This suggests that our ergometer provides a suitable emulation of bicycle lean dynamics. Overall, we find that leaning a cycle ergometer ad libitum does not enhance maximal power output, and conversely, trying to minimize lean impairs maximal power output.
\end{abstract}

Keywords: human power, lateral sway, stability

\section{Introduction}

Competitive cyclists sprint out of the saddle and alternately lean their bikes from side-to-side, away from the downstroke pedal (Soden \& Adeyefa, 1979). Cyclists (TrainerRoad, 2018), coaches (Forbes, n.d.), and journalists (CyclingTips, 2009) opine that leaning the bicycle improves sprint performance. But, some scientists advise that bicycle lean can impair performance (Bouillod et al. 2018). In their words, "cyclists should decrease bicycle sways". They base this advice on the assertion that lean increases rolling

*Corresponding author. Email: ross.wilkinson@colorado.edu All authors have read and approved this version of the manuscript for pre-print. Authors RDW@rd_wilkinson and RK@RodgerKram can be reached on Twitter.

Please cite as: Wilkinson, R.D., \& Kram R. (2021). The influence of bicycle lean on maximal power output during sprint cycling. SportR $\chi$ iv. https://doi.org/10.31236/osf.io/xa7th

Preprint submitted to Journal of Biomechanics

February 23, 2021 
resistance due to tire deformation. If lean does increase rolling resistance, then indeed minimizing lean might enhance sprint performance. But only if minimizing lean does not substantially reduce power output. To date, there is no direct evidence that leaning or attempting to minimize lean affects maximal power output during sprint cycling.

Scientists often measure a cyclist's maximal power on a traditional stationary ergometer (e.g. Reiser et al. (2002); Dorel et al. (2005); Gardner et al. (2007); Hug et al. (2011); Merkes et al. (2020)). But unlike a bicycle, ergometers typically cannot lean from side-to-side. A stationary ergometer removes worry about stability and may allow a rider to sprint with abandon. In contrast, during overground cycling sprints, riders use their arms to stabilize and actively lean the bicycle. These differences have limited our understanding of how stability and bicycle lean affect maximal power during sprint cycling. Gardner et al. (2007) compared maximal power output on a traditional stationary ergometer versus overground cycling. They allowed their subjects to lean their bicycles ad libitum and found that maximal power was similar between the two conditions. But, their results may have been affected by the difference in inertial load between the two conditions.

Further, the question of whether minimizing lean affects maximal power remains open. Wilkinson et al. (2020) compared rider biomechanics on rollers, but only at a submaximal power output $\left(5 \mathrm{~W} \mathrm{~kg}^{-1}\right)$. Subjects rode in a non-seated posture, while either leaning the bicycle ad libitum or under instruction to minimize lean. In the ad libitum lean condition, riders produced higher peak vertical crank force, raised and lowered their center of mass to a greater extent, and increased the effective mechanical advantage of their hip and knee extensor muscles. These differences in rider biomechanics may also occur when trying to minimize lean during an all-out sprint.

Here, we modified a cycle ergometer so that it could lean from side-to-side or be locked to prevent lean. This allows riders to sprint under three different conditions: locked (no lean), ad libitum lean, and minimal lean. In the minimal lean condition, the ergometer could lean but we asked the subjects to try to minimize lean. Our first hypothesis (null) was that ad libitum lean would result in the same maximal power as the locked condition. Our second hypothesis was that trying to minimize lean would decrease maximal power compared to the $a d$-lib and locked conditions. We further hypothesized that in the $a d$-lib condition, riders would use their arms to lean the ergometer away from the downstroke pedal - as in normal overground cycling.

\section{Materials and methods}

\subsection{Experimental design}

Nineteen healthy recreational cyclists volunteered (13 men/6 women, age: $27.6 \pm 6.2$ years, height: $1.75 \pm 0.09 \mathrm{~m}$, mass: $68.9 \pm 10.4 \mathrm{~kg}$, mean $\pm \mathrm{SD})$. Subjects were between 18-49 years old, in good health, cycled $>4$ hours per week, and used their own clip-in pedals and shoes. All subjects gave written informed consent as per the University of Colorado Boulder Institutional Review Board.

Subjects pedaled a friction-loaded ergometer (Nobilette, Longmont, CO, USA) equipped with a crank-based mechanical power meter (Quarq DZero, SRAM, Corp, Chicago, IL, USA) with $172.5 \mathrm{~mm}$ long cranks. We matched the saddle and handlebar positions of the ergometer to the subject's own road bicycle. 
To create frictional resistance, we wrapped a $4 \mathrm{~mm}$ diameter polyester rope around the circumference of the ergometer flywheel. The flywheel is a standard Monark aluminum model $827 \mathrm{E}$ with a diameter of $0.26 \mathrm{~m}$ and a mass of $20 \mathrm{~kg}$ (Monark $\mathrm{AB}$, Vansbro, Sweden). We fixed one end of the rope to the ergometer frame and hung the other end over a wall-mounted pulley. This allowed us to adjust the resistance on the flywheel by hanging different weights from the rope. The height of the wall-mounted pulley was level with the top of the flywheel which ensured that the horizontal force in the rope was equal to the hanging weight. Because leaning the ergometer creates a lateral displacement of the rope, we positioned the ergometer $3 \mathrm{~m}$ away from the pulley to minimize fluctuations in resistance due to geometry (see Figure $1 \mathrm{~A}$ ).

A crank-mounted sensor (Garmin Ltd, Olathe, KA, USA) measured cadence with 1 $\mathrm{Hz}$ resolution. The power meter creates a 1-s average from data measured at $65 \mathrm{~Hz}$. A cycling computer mounted to the handlebar (Edge 1000, Garmin Ltd, Olathe, KA, USA) recorded the cadence and crank power. As per the manufacturer's recommendations, we zeroed the power meter prior to each testing session.

We mounted the ergometer frame atop a hinged chassis that allowed rotation in the frontal plane. In the $a d$-lib lean and minimal lean conditions, springs attached to the rear legs of the ergometer provided a restoring torque proportional to lean angle. We tuned the spring mechanism during pilot testing to emulate the lateral dynamics of a bicycle. The final design included four, $15 \mathrm{~cm}$ long, steel extension springs in parallel on each side of the ergometer. Each spring had a spring constant of $771 \mathrm{~N} \mathrm{~m}^{-1}$. Together, the springs provided a restoring torque of $7.6 \mathrm{~N} \mathrm{~m}$ per degree of ergometer rotation. In frontal plane rotation, the system is an under-damped simple harmonic oscillator. To prevent movement in the frontal plane during the locked condition, aluminum struts braced the chassis pivot.

Subjects warmed up by cycling in a seated posture at a low intensity $(\sim 100$ watts $)$ for $5 \mathrm{~min}$. This was followed by three to five short (5-s) maximal sprints in a nonseated posture under the ad-lib lean condition, each separated by 3-min of rest. These warm-up sprints familiarized subjects to the side-to-side movement of the ergometer. We also used these warm-up sprints to individualize the resistance of the ergometer to ensure a valid maximal power result. We deemed a subject's maximal power to be valid if the maximum cadence during the sprint was between 90-145 rpm (Driss \& Vandewalle, 2013). Thus, we adjusted the hanging weight for each warm-up sprint based on the maximum cadence during the prior sprint. We set the initial hanging weight and gradations as percentages of the subject's body weight because of the strong correlation between bodyweight and maximal crank power (Stone \& Hull, 1995). We increased the hanging weight until there was a noticeable drop in the subject's maximal power. This generated a subject-specific weight-power-cadence relationship, which we used to estimate their optimal hanging weight (see Figure 1 B). Our estimation used a $2^{\text {nd }}$-order polynomial to model the subject's maximal power as a function of hanging weight.

Prior to the experimental trials, subjects rested for $10 \mathrm{~min}$. For each experimental trial, subjects completed a maximal 5-s sprint from rest in a non-seated posture. Subjects performed 9 experimental sprints - 3 locked, 3 ad-lib lean, and 3 minimal lean-with 3min rest between each. We split the 9 sprints into 3 blocks of 3 sprints and randomized the order of conditions - without repetition - within each block. We also ensured that subjects did not repeat the same order of conditions between blocks. 


\subsection{Data analysis}

We calculated crank torque at maximal power by dividing the recorded maximal 1 -s crank power in $\mathrm{W}$ by the cadence at maximal power in $\mathrm{rad} \mathrm{s}^{-1}$. Linear regression analyses determined the torque $v s$. hanging weight relationship for each subject using the maximum 1-s crank power and cadence values recorded in each of the warm-up sprints (see Figure 1C).

We derived instantaneous crank angle and ergometer lean angle using two Inertial Measurement Units (IMU) (BlueThunder Sensor, IMeasureU, Auckland, New Zealand) - one secured using double-sided tape to the left crank and another to the seat tube. We used the IMU Research Application (IMeasureU, Auckland, New Zealand) to record 3 -axis accelerometer data at $500 \mathrm{~Hz}$. At the beginning of each sprint trial, we lightly tapped both IMU sensors simultaneously while starting the cycle computer. This created an artificial spike in each IMUs resultant acceleration, enabling us to subsequently synchronize the cycling computer and IMU data.

We imported the raw IMU data files into MATLAB (R2020a, The MathWorks Inc., Natick, MA, USA) and processed them using custom scripts. We low-pass filtered (2 $\mathrm{Hz}$ cutoff, minimum-order Butterworth) the acceleration signals from both IMUs. The timing of peak acceleration in the global z-axis (i.e. vertical) measured by the IMU on the left crank created an event signal when the IMU passed bottom dead center, i.e. when the right crank passed top dead center. To calculate lean angle, we used the measured acceleration in the global y-axis (medio-lateral) of the IMU on the ergometer frame. We converted the signal to angular acceleration using the known height of the IMU above the ergometer pivot, differentiating twice to get angular displacement, and then detrended the displacement values. We verified the accuracy of this method by performing static and dynamic trials with the ergometer set lean angles of $10^{\circ}$. Finally, we parsed the lean angle signal between each top dead center location and calculated the range (max-min) of lean during each crank cycle.

\subsection{Statistical analysis}

We used a one-way repeated-measures analysis of variance (ANOVA) to compare key variables between conditions. For each outcome measure, we used each subject's mean from the three replicates within each condition. Performing three replicates allowed each subject to regress to their mean within each condition. The assumption of taking a mean of the replicates is that of equal variance within each condition and each subject. We used Bartlett's test to verify that assumption. An a priori power analysis $\left(\mathrm{G}^{*}\right.$ Power v3.1), determined that a sample size of 19 would be required to detect our smallest effect size of interest (0.48) and that we could detect effect sizes $\geq 0.8$ with $90 \%$ power at $\alpha=0.05$. We corrected p-values for multiple comparisons using the Dunn-Sidak method. For multiple comparisons, we provide the effect size (ES) as Hedges' $\mathrm{g}_{\mathrm{av}}$. We present the descriptive data for the initial sprint testing results as the group mean \pm one standard deviation, whereas the results from each experimental condition are the group mean \pm the pooled standard deviation. 


\section{Results}

\subsection{Relations of power output, cadence, and crank torque to hanging weight}

The average predicted optimal hanging weight across our subject group was $12 \pm 2 \%$ of bodyweight (see Figure 1D) corresponding to maximal 1-s crank power of $927 \pm 200$ $\mathrm{W}\left(13.5 \pm 2.2 \mathrm{~W} \mathrm{~kg}^{-1}\right)$ at a cadence of $111 \pm 10 \mathrm{rpm}$ (see Figure $1 \mathrm{E}$ ) and a crank torque of $80 \pm 18 \mathrm{~N}$ m (see Figure $1 \mathrm{~F}$ ).

[Figure 1 about here.]

\subsection{Maximal 1-s crank power}

Maximal 1-s crank power results are presented in Figure 2A. Maximal power in the ad-lib condition $(974 \pm 33 \mathrm{~W})$ was similar to the locked condition $(982 \pm 30 \mathrm{~W}), \mathrm{t}=0.8$, $\mathrm{p}=0.8, \mathrm{CI}_{95 \%}[-34,18], \mathrm{ES}=0.2$, but the minimal lean condition was $47 \mathrm{~W}(5 \%)$ less $(927 \pm 32 \mathrm{~W}), \mathrm{t}=5.5, \mathrm{p}<0.001, \mathrm{CI}_{95 \%}[25,69], \mathrm{ES}=1.3$. Maximal power was $55 \mathrm{~W}$ $(6 \%)$ lower in the minimal lean condition compared to locked, $\mathrm{t}=7.6, \mathrm{p}<0.001, \mathrm{CI}_{95 \%}$ $[-74,-36], \mathrm{ES}=1.6$.

\subsection{Range and pattern of ergometer lean}

Ergometer lean angles are presented in Figure 2 3 . The range of lean in the $a d$ lib condition $\left(9.6 \pm 2.3^{\circ}\right)$ was an order of magnitude greater than the locked condition $\left(0.8 \pm 0.3^{\circ}\right), \mathrm{t}=6.7, \mathrm{p}<0.001, \mathrm{CI}_{95 \%}[5.0,11.8], \mathrm{ES}=1.7$, and more than twice the minimal lean condition $\left(3.9 \pm 1.0^{\circ}\right), \mathrm{t}=5.2, \mathrm{p}<0.001, \mathrm{CI}_{95 \%}[2.6,8.5], \mathrm{ES}=1.3$. The range of lean in the minimal lean condition was four times greater than in the locked condition, $\mathrm{t}=6.7, \mathrm{p}<0.001, \mathrm{CI}_{95 \%}[1.7,4.0], \mathrm{ES}=1.7$. The patterns of lean across the crank cycle in each condition are depicted in Figure 3 . During the ad-lib condition, a noticeable counterclockwise rotation - away from the downstroke pedal - of the ergometer occurred when the right crank reached bottom dead center (BDC, $180^{\circ}$ ) and a clockwise rotation occurred when the left crank reached BDC.

\subsection{Cadence}

Cadence values at maximal power are presented in Figure $2 \mathrm{C}$. There were no conclusive differences in cadence at maximal power between the ad-lib condition $(111 \pm 5 \mathrm{rpm})$ compared to the locked condition $(114 \pm 4 \mathrm{rpm}), \mathrm{t}=2.2, \mathrm{p}=0.117, \mathrm{CI}_{95 \%}[-4.7,0.4]$, $\mathrm{ES}=0.5$, nor the minimal lean condition $(109 \pm 3 \mathrm{rpm}), \mathrm{t}=2.5, \mathrm{p}=0.062, \mathrm{CI}_{95 \%}[-0.1$, $5.0], \mathrm{ES}=0.6$. Cadence at maximal power in the minimal lean condition averaged 5 $\mathrm{rpm}(4 \%)$ slower than in the locked condition, $\mathrm{t}=8.1, \mathrm{p}<0.001, \mathrm{CI}_{95 \%}[-6.1,-3.1]$, $\mathrm{ES}=1.7$.

\subsection{Crank torque}

Crank torque data at maximal power are presented in Figure 2D. Crank torque at maximal power in the ad-lib condition $(83.7 \pm 18.3 \mathrm{~N} \mathrm{~m})$ was similar to the locked condition $(82.8 \pm 18.9 \mathrm{~N} \mathrm{~m}), \mathrm{t}=1.7, \mathrm{p}=0.304, \mathrm{CI}_{95 \%}[-0.5,2.3], \mathrm{ES}=0.4$, but $2.2 \mathrm{~N}$ $\mathrm{m}(3 \%)$ greater than the minimal lean condition $(81.5 \pm 19.2 \mathrm{~N} \mathrm{~m}), \mathrm{t}=3.3, \mathrm{p}=0.013$, $\mathrm{CI}_{95 \%}[0.4,4.0], \mathrm{ES}=0.7$. The difference in crank torque at maximal power between the minimal lean and locked conditions was inconclusive, $\mathrm{t}=2.3, \mathrm{p}=0.09, \mathrm{CI}_{95 \%}[-2.7$, $0.2], \mathrm{ES}=0.5$. 
[Figure 2 about here.]

[Figure 3 about here.]

\section{Discussion}

We accept our first two hypotheses; the ability to lean the ergometer did not increase maximal power compared to a traditional stationary ergometer and maximal power decreased (by 5\%) when attempting to minimize lean compared to when leaning ad libitum or locked. The similarity in maximal power between the ad-lib and locked condition is consistent with the findings of Gardner et al. (2007) who found no difference in maximal power during non-seated sprint cycling on a traditional stationary ergometer compared to overground cycling in a group of elite track cyclists.

It appears that during overground cycling, bicycle lean provides a sufficient level of lateral stability for riders to simultaneously generate maximal power and maintain dynamic balance. We propose that leaning the bicycle away from the downstroke pedal allows riders to utilize the angular momentum of the bicycle - the product of bicycle moment of inertia and lean angular velocity - to offset a portion of the angular impulse - the time integral of torque around the relevant axis of rotation - around the longitudinal axis of the bicycle. When a rider tries to minimize bicycle lean and thus angular momentum of the bicycle, then they must offset this angular impulse by either pulling harder on the contralateral handlebar or by reducing the force they exert on the crank. The disadvantage of decreasing crank force in a sprint is obvious. Thus, the choice becomes whether to use the arms to pull harder on the handlebars or to lean the bicycle. The widespread use of bicycle lean suggests that it is the optimal strategy, but further analyses are needed.

Our IMU-derived data for the pattern of ergometer lean support our third hypothesis; during the $a d$-lib condition subjects leaned the ergometer away from the downstroke pedal as in overground cycling. The pattern and magnitude of ergometer lean during the $a d-l i b$ condition was in general agreement with previous reports of bicycle lean when cycling overground (Soden \& Adeyefa, 1979), on a treadmill (Hull et al., 1990), and on rollers (Wilkinson et al., 2020). This suggests that our modified cycle ergometer provided a suitable emulation of bicycle lean dynamics.

What explains the decline in maximal power when attempting to minimize lean? Wilkinson et al. (2020) found that attempting to minimize bicycle lean during submaximal cycling decreases a rider's vertical CoM movement and crank force production. It follows that riders in our study reduced their vertical CoM displacement, which may have decreased the non-muscular contribution to crank force and power. Future studies on overground sprint cycling could test this idea by placing a single IMU on the rider's lower back to estimate the vertical motion and total mechanical energy fluctuations of the rider's CoM under different lean conditions (Wilkinson \& Lichtwark, 2020).

There is strong evidence from previous research on resistance training (Drinkwater et al., 2007) and rowing (Sarabon et al., 2019) that unstable environments can impair performance. Furthermore, it has been suggested that such decrements are associated with a joint stiffening strategy when there is a greater threat to dynamic balance (Adkin et al. 2002, Carpenter et al., 2001). It appears that the stability provided by either the locked ergometer or leaning the bicycle ad libitum are sufficient for riders to generate 
their maximal 1-s crank power. Attempting to minimize lean when no lateral support is provided appears to be unstable and clearly inhibits power production.

Our results may not generalize to maximal power production during overground cycling. Lateral bicycle dynamics, friction, tire deformation, aerodynamics, and visual stimuli may all confound the ecological validity of our findings. However, the similarity in lean pattern used in the $a d$-lib condition to overground and treadmill cycling suggests that the dynamics were suitably emulated. We discount the idea that cyclists should avoid leaning the bicycle due to a possible increase in tire deformation and hence rolling resistance (Bouillod et al., 2018). A bicycle-rider system weighing $80 \mathrm{~kg}$ travelling at 65 $\mathrm{km} \mathrm{hr}^{-1}$ requires a total mechanical power output of $\sim 1000 \mathrm{~W}$ and rolling resistance comprises only $4.5 \%$ of total power dissipation ( $45 \mathrm{~W}$ ) (Martin et al. 1998). We found that trying to avoid lean reduced mechanical power output by $5 \%$. Thus, avoiding lean in this scenario would result in a net power deficit unless leaning increased the power dissipation due to rolling resistance to $>95 \mathrm{~W}$, which would require a $100 \%$ increase in the coefficient of rolling resistance.

It is also possible that our results may not generalize to elite sprint cyclists. The decrease in maximal power when attempting to minimize lean may only be a characteristic of untrained or non-competitive cyclists, as the maximal power of our subject group $\left(982 \pm 245 \mathrm{~W}, 14.3 \mathrm{~W} \mathrm{~kg}^{-1}\right)$ was well below that reported for elite track cyclists $\left(1791 \pm 169 \mathrm{~W}, 20.8 \mathrm{~W} \mathrm{~kg}^{-1}\right)$ (Gardner et al. 2007). However, we believe the heterogeneity of our subject group provides strong evidence that the effects of bicycle lean likely generalize to riders of all abilities. For example, maximal power ranged from 656 to 1412 $\mathrm{W}$, yet maximal power decreased for every subject when attempting to minimize lean. Furthermore, it is possible that with enough training, our subjects may have been able to produce greater power when attempting to minimize lean.

Finally, small differences in maximal power may exist between the $a d-l i b$ lean and locked conditions, but were too small to detect in this study. This study was sufficiently powered to detect effect sizes $\geq 0.48$, but the effect size between ad-lib lean and locked was only $0.2(\sim 8 \mathrm{~W}, 0.8 \%)(\mathrm{p}=0.8)$.

In conclusion, we find that maximal 1-s crank power during non-seated, sprint cycling is similar when leaning the bicycle ad libitum compared to a traditional stationary ergometer. Trying to minimize bicycle lean decreases maximal 1-s crank power by an average of $5 \%$ compared to leaning ad libitum. Similar to overground cycling, riders leaned the modified ergometer away from the downstroke pedal, which suggests that our ergometer provides a suitable emulation of bicycle lean dynamics.

\section{Conflict of interest statement}

Our laboratory is financially supported by Specialized Bicycle Components Inc. but they did not commission or fund this specific study.

\section{Acknowledgements}

This research was supported by an International Society of Biomechanics Student International Travel Grant awarded to RDW. We thank Prof. William Byrnes for the loan of the ergometer. 


\section{References}

Adkin, A. L., Frank, J. S., Carpenter, M. G., \& Peysar, G. W. (2002). Fear of falling modifies anticipatory postural control. Experimental Brain Research, 143, 160-170. doi 10.1007/s00221-001-0974-8

Bouillod, A., Pinot, J., Valade, A., Cassirame, J., Soto-Romero, G., \& Grappe, F. (2018). Influence of standing position on mechanical and energy costs in uphill cycling. Journal of Biomechanics, 72, 99-105. doi 10.1016/j.jbiomech.2018.02.034

Carpenter, M. G., Frank, J. S., Silcher, C. P., \& Peysar, G. W. (2001). The influence of postural threat on the control of upright stance. Experimental Brain Research, 138, 210-218. doi 10.1007/ s002210100681

CyclingTips (2009). Cav's Perfect Sprint Technique. URL: https://cyclingtips.com/2009/07/ cavs-perfect-sprint-technique/.

Dorel, S., Hautier, C. A., Rambaud, O., Rouffet, D., Van Praagh, E., Lacour, J. R., \& Bourdin, M. (2005). Torque and power-velocity relationships in cycling: Relevance to track sprint performance in worldclass cyclists. International Journal of Sports Medicine, 26, 739-746. doi 10.1055/s-2004-830493

Drinkwater, E. J., Pritchett, E. J., \& Behm, D. G. (2007). Effect of instability and resistance on unintentional squat-lifting kinetics. International journal of sports physiology and performance, 2, 400-413. doi 10.1123/ijspp.2.4.400

Driss, T., \& Vandewalle, H. (2013). The Measurement of Maximal (Anaerobic) Power Output on a Cycle Ergometer: A Critical Review. BioMed Research International, 2013, 1-40. doi 10.1155/ 2013/589361

Forbes, O. (n.d.). The Four Phases Of Sprint Training." [online] Trainingpeaks.com. URL: https: //www.trainingpeaks.com/blog/the-four-phases-of-sprint-training/

Gardner, A. S., Martin, J. C., Martin, D. T., Barras, M., \& Jenkins, D. G. (2007). Maximal torqueand power-pedaling rate relationships for elite sprint cyclists in laboratory and field tests. European Journal of Applied Physiology, 101, 287-292. doi 10.1007/s00421-007-0498-4

Hug, F., Turpin, N. A., Couturier, A., \& Dorel, S. (2011). Consistency of muscle synergies during pedaling across different mechanical constraints. Journal of neurophysiology, 106, 91-103. doi 10. 1152/jn.01096.2010

Hull, M. L., Beard, A., \& Varma, H. (1990). Goniometric measurement of hip motion in cycling while standing. Journal of Biomechanics, 23, 687-703.

Martin, J. C., Milliken, D. L., Cobb, J. E., McFadden, K. L., \& Coggan, A. R. (1998). Validation of a mathematical model for road cycling power. Journal of Applied Biomechanics, 14, 276-291.

Merkes, P. F., Menaspà, P., \& Abbiss, C. R. (2020). Power output, cadence, and torque are similar between the forward standing and traditional sprint cycling positions. Scandinavian Journal of Medicine and Science in Sports, 30, 64-73.

Reiser, R. F., Maines, J. M., Eisenmann, J. C., \& Wilkinson, J. G. (2002). Standing and seated Wingate protocols in human cycling. A comparison of standard parameters. European Journal of Applied Physiology, 88, 152-157. doi 10.1007/s00421-002-0694-1

Sarabon, N., Kozinc, Z., Babic, J., \& Markovic, G. (2019). Effect of rowing ergometer compliance on biomechanical and physiological indicators during simulated 2,000-metre race. Journal of Sports Sciences, 18, 264-270.

Soden, P., \& Adeyefa, B. A. (1979). Forces applied to a bicycle during normal cycling. Journal of Biomechanics, 12, 527-541.

Stone, C., \& Hull, M. L. (1995). The effect of rider weight on rider-induced loads during common cycling situations. Journal of Biomechanics, 28, 365-375. doi 10.1016/0021-9290(94)00102-A

TrainerRoad (2018). Sprinting Tips From A Pro - Team Clif Bar Racing's Pete Morris. [video]. URL: https://www youtube. com/watch?v=q1ZAACbDxiA

Wilkinson, R. D., Cresswell, A. G., \& Lichtwark, G. A. (2020). Rock and roll : The influence of bicycle lean on the mechanics of non-seated cycling. SportRxiv, August 27. doi http://dx.doi.org/ 10.31236/osf.io/hj9gp

Wilkinson, R. D., \& Lichtwark, G. A. (2020). A method for tracking centre of mass displacement during non-seated cycling using an inertial sensor. SportRxiv, August 27. doi http://dx.doi.org/10.31236/ osf.io/htr $4 \mathrm{k}$ 


\section{List of Figures}

1 Relations of power output, cadence, and crank torque to hanging weight . 10

2 Individual and group mean results $\ldots \ldots \ldots \ldots \ldots$

$3 \quad$ Ergometer lean patterns $\ldots \ldots \ldots \ldots \ldots$. . . . . . . . . . . . . . 12 
A
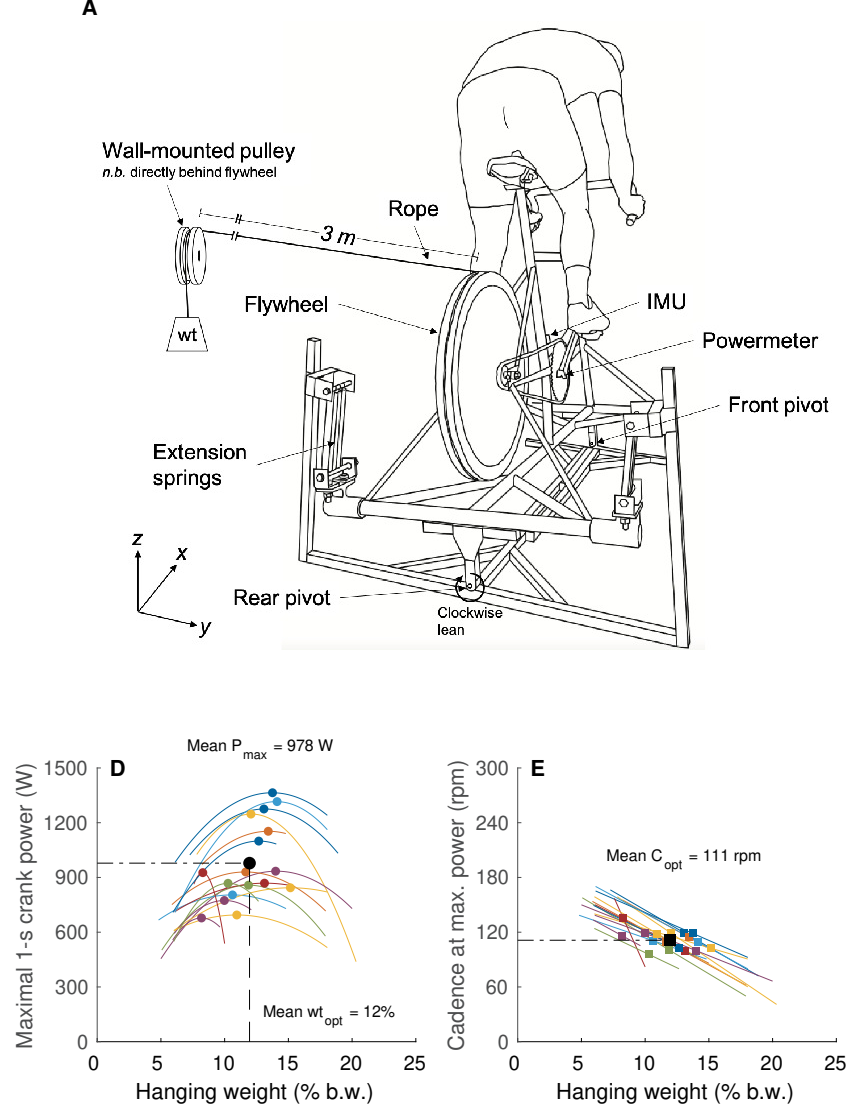
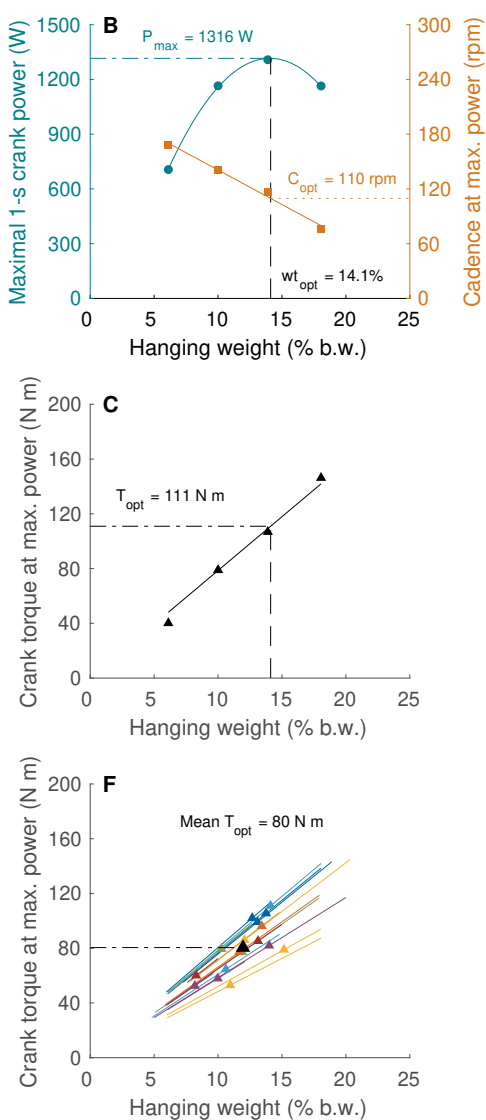

Figure 1: A. Modified cycle ergometer which can either constrain or allow side-to-side lean in the frontal plane. The springs provide a restoring torque proportional to lean angle. The pulley system provides frictional resistance proportional to the hanging weight. B. An example of how we determined the hanging weight that would elicit maximal power $\left(\mathrm{P}_{\max }\right)$ for each subject $\left(\mathrm{wt}_{\mathrm{opt}}\right)$, prior to conducting the experimental trials. We fit a $2^{\text {nd }}$-order polynomial to maximal power against a variety of hanging weights $\left(\mathrm{R}^{2}>0.99\right)$ to determine $\mathrm{P}_{\max }$ and the associated $\mathrm{wt}_{\mathrm{opt}}$. Additionally, we fit a linear model to the cadence achieved at maximal power $\left(\mathrm{R}^{2}=0.99\right)$ to confirm that the subject's predicted cadence at maximal power fell within population norms (90 to $145 \mathrm{rpm}$ (Driss \& Vandewalle, 2013). C. The strong positive relationship between crank torque and hanging weight - as shown by the linear regression $\left(\mathrm{R}^{2}=0.99\right)$ - exists because the two are linked by the mechanical advantage of the ergometer's drivetrain. Thus, the strength and direction of the relationships that power and cadence share with crank torque are mimicked by hanging weight. D. Power-hanging weight relationships determined for each of the 19 subjects $\left(\right.$ Mean $\mathrm{R}^{2}=0.98$ ). wt $\mathrm{w}_{\text {opt }}$ values ranged between $8.2-15.2 \%$ b.w. Predicted $\mathrm{P}_{\max }$ values ranged between 678-1364 W. Individual and group mean values for $\mathrm{P}_{\max }$ and $\mathrm{wt}_{\mathrm{opt}}$ are indicated by color and black squares, respectively. E. Cadence-hanging weight relationships determined for each subject (Mean $\mathrm{R}^{2}=0.97$ ). $\mathrm{C}_{\text {opt }}$ values ranged between 96-135 rpm. Individual and group mean $\mathrm{C}_{\text {opt }}$ values are indicated by color and black squares, respectively. F. Crank torque-hanging weight relationships determined for each subject $\left(\right.$ Mean $\mathrm{R}^{2}=0.9$ ). Predicted $\mathrm{T}_{\mathrm{opt}}$ values ranged between $52-111 \mathrm{~N} \mathrm{~m}$. Individual and group mean $\mathrm{T}_{\mathrm{opt}}$ values are indicated by color and black triangles, respectively. 

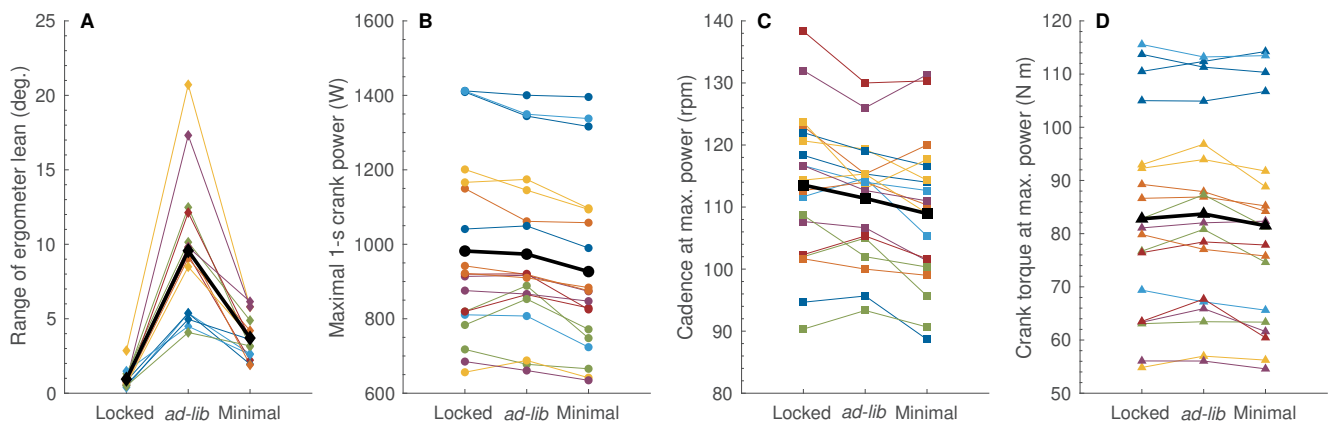

Figure 2: A. Individual mean (color) and group mean (black) range (peak-to-peak) of ergometer lean during maximal sprints in each condition. On average, the range of lean increased significantly in $a d-l i b$ compared to the locked $(\mathrm{p}<0.001)$ and minimal lean $(\mathrm{p}<0.001)$ conditions. B. Individual mean (color) and group mean (black) maximal 1-s crank power. Maximal 1-s crank power was similar between $a d-l i b$ and locked $(\mathrm{p}=0.8)$, but decreased by $47 \mathrm{~W}(5 \%, \mathrm{p}<0.001)$ and $55 \mathrm{~W}(6 \%, \mathrm{p}<0.001)$ in the minimal lean condition compared to $a d$-lib and Locked, respectively. C. Individual mean (color) and group mean (black) cadence at maximal power. The cadence at maximal power was similar between the $a d-l i b$ and locked conditions $(\mathrm{p}=0.117)$, however cadence decreased by $\sim 4 \%$ in the minimal lean condition compared to locked ( $\mathrm{p}<0.001)$. D. Individual mean (color) and group mean (black) crank torque at maximal power. Crank torque at maximal power was similar between the locked and minimal lean conditions $(\mathrm{p}=0.304)$, but crank torque decreased by $\sim 3 \%$ in the minimal lean condition compared to $a d-l i b(\mathrm{p}=0.013)$. 

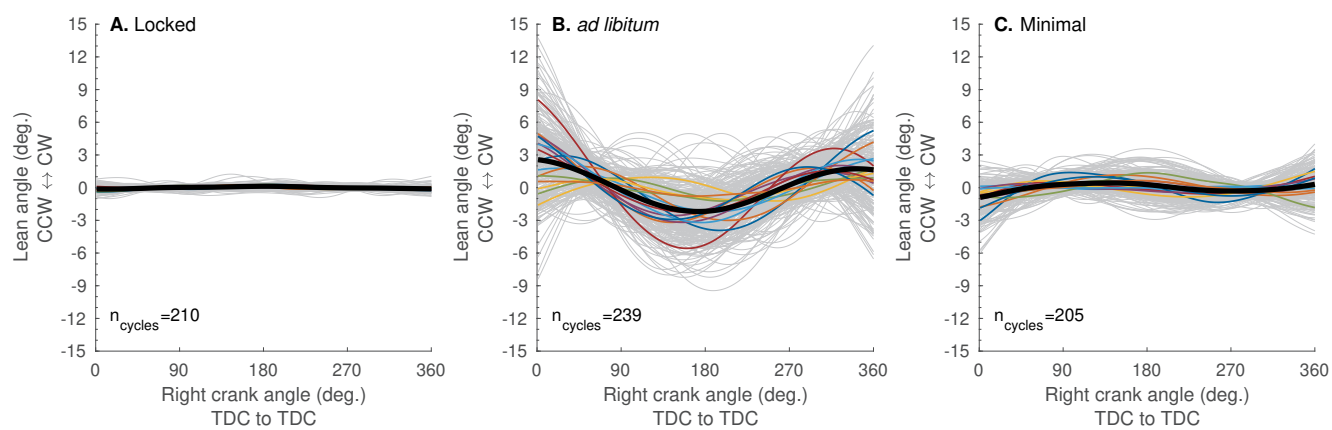

Figure 3: Ergometer lean angles from all crank cycles and all subjects $(\mathrm{n}=19)$ are shown by the light grey lines. The individual means are shown in color and the group mean in black. Note that temporal variability in peak lean angle between crank cycles within a trial results in lower average amplitudes of lean for each individual compared to their respective mean range shown in Figure 2 (i.e. range of mean $v s$. mean of range). Positive values indicate a clockwise rotation when viewing the ergometer from the rear. $\mathrm{n}_{\text {cycles }}$, total number of crank cycles analyzed across all subjects within each condition. 